九州大学学術情報リポジトリ

Kyushu University Institutional Repository

\title{
On Modular Forms Arising from a Differential Equation of Hypergeometric Type
}

Kaneko, Masanobu

Graduate School of Mathematics, Kyushu University

Koike, Masao

Graduate School of Mathematics, Kyushu University

http://hdl. hand le. net/2324/20434

出版情報: The Ramanujan Journal. 7 (1-3), pp.145-164, 2003. Springer バージョン：

権利関係 : 


\title{
ON MODULAR FORMS ARISING FROM A DIFFERENTIAL EQUATION OF HYPERGEOMETRIC TYPE
}

\author{
Masanobu Kaneko and Masao Koike
}

\section{$\S 1$. INTRODUCTION}

The differential equation that the present paper shall deal with is

$$
(\#)_{k} \quad f^{\prime \prime}(\tau)-\frac{k+1}{6} E_{2}(\tau) f^{\prime}(\tau)+\frac{k(k+1)}{12} E_{2}^{\prime}(\tau) f(\tau)=0,
$$

where $\tau$ is a variable in the upper half-plane $\mathfrak{H},^{\prime}$ a differential operator $(2 \pi i)^{-1} d / d \tau=$ $q \cdot q / d q\left(q=e^{2 \pi i \tau}\right)$, and $E_{2}(\tau)$ the "quasimodular" Eisenstein series of weight 2 for the full modular group $\mathrm{SL}_{2}(\mathbb{Z})$ :

$$
E_{2}(\tau)=1-24 \sum_{n=1}^{\infty}\left(\sum_{d \mid n} d\right) q^{n}
$$

The parameter $k$ always stands for a non-negative integer or half an integer throughout the paper. This differential equation originates in the work [1] where in some cases $(k \equiv 0,4 \bmod 6)$ solutions which are modular on $\mathrm{SL}_{2}(\mathbb{Z})$ were found and studied in connection with liftings of supersingular $j$-invariants of elliptic curves.

The purpose of this paper is to give an explicit description of (conjecturally) all modular solutions of $(\#)_{k}$ when $k$ is an integer or half an integer (Theorem 1 in Section 2), as well as to discuss positiveness of Fourier coefficients of some of those solutions (Theorem 3 in Section 4). We shall also discuss an intrinsic characterization of the equation $(\#)_{k}$ by the property that if $f(\tau)$ is a solution, then $(c \tau+d)^{-k} f((a \tau+b) /(c \tau+d))$ is also a solution for all $\left(\begin{array}{ll}a & b \\ c & d\end{array}\right) \in \mathrm{SL}_{2}(\mathbb{Z})$ (Proposition 2 in Section 5). When $k$ is an odd integer congruent to 5 modulo 6 , an unexpected solution occurs in contrast to the other cases: $(\#)_{k}$ has quasimodular solutions of weight $k+1$ (rather than $k$ as in other modular solutions). We shall describe this quasimodular solution in terms of certain orthogonal polynomials (Theorem 2 in Section 2), and, to show that this is a solution, discuss an inductive structure of solutions of $(\#)_{k}$ with different $k$ 's in Section 3.

\section{§2. Explicit DEscription of modular and quasimodular solutions}

To describe the solutions, we need to develop notations of various modular forms of levels $1,2,3$, and 4 . Let

$$
E_{4}(\tau)=1+240 \sum_{n=1}^{\infty}\left(\sum_{d \mid n} d^{3}\right) q^{n}=1+240 q+2160 q^{2}+6720 q^{3}+\cdots
$$


and

$$
E_{6}(\tau)=1-504 \sum_{n=1}^{\infty}\left(\sum_{d \mid n} d^{5}\right) q^{n}=1-504 q-16632 q^{2}-122976 q^{3}-\cdots
$$

be the Eisenstein series of weight 4 and 6 on $\mathrm{SL}_{2}(\mathbb{Z})$,

$$
\Delta(\tau)=\frac{E_{4}(\tau)^{3}-E_{6}(\tau)^{2}}{1728}=q-24 q^{2}+252 q^{3}-1472 q^{4}+\cdots
$$

the "discriminant" cusp form of weight 12 and

$$
j(\tau)=\frac{E_{4}(\tau)^{3}}{\Delta(\tau)}=\frac{1}{q}+744+196884 q+21493760 q^{2}+\cdots
$$

the modular invariant. For an integer $N$, let $\Gamma_{0}(N)$ denote the modular group of level $N$ defined by

$$
\Gamma_{0}(N)=\left\{\left(\begin{array}{ll}
a & b \\
c & d
\end{array}\right) \in \mathrm{SL}_{2}(\mathbb{Z}) \mid c \equiv 0 \bmod N\right\} .
$$

Define

$$
\begin{aligned}
E_{2}^{(2)}(\tau) & :=2 E_{2}(2 \tau)-E_{2}(\tau) \\
& =1+24 \sum_{n=1}^{\infty}\left(\sum_{\substack{d \mid n \\
d: \text { odd }}} d\right) q^{n}=1+24 q+24 q^{2}+96 q^{3}+\cdots \\
\Delta_{4}^{(2)}(\tau) & :=\frac{\eta(2 \tau)^{16}}{\eta(\tau)^{8}}=\sum_{n=1}^{\infty}\left(\sum_{\substack{d \mid n \\
d: \text { odd }}}(n / d)^{3}\right) q^{n}=q+8 q^{2}+28 q^{3}+64 q^{4}+\cdots \\
j^{(2)}(\tau) & :=\frac{E_{2}^{(2)}(\tau)^{2}}{\Delta_{4}^{(2)}(\tau)}=\frac{1}{q}+40+276 q-2048 q^{2}+\cdots
\end{aligned}
$$

where

$$
\eta(\tau)=q^{\frac{1}{24}} \prod_{n=1}^{\infty}\left(1-q^{n}\right)=q^{\frac{1}{24}}-q^{\frac{25}{24}}-q^{\frac{49}{24}}+q^{\frac{121}{24}}+\cdots
$$

is the Dedekind eta function. The functions $E_{2}^{(2)}(\tau)$ and $\Delta_{4}^{(2)}(\tau)$ are modular forms of respective weights 2 and 4 on the group $\Gamma_{0}(2)$, and $j^{(2)}(\tau)$ is a $\Gamma_{0}(2)$-invariant function which generates the field of modular functions on $\Gamma_{0}(2)$ (the normalized function $j^{(2)}(\tau)-40$ is often referred to as the "Hauptmodul" for the group $\Gamma_{0}(2)$ ). In addition, the function

$$
\sqrt{\Delta_{4}^{(2)}(\tau)}=\frac{\eta(2 \tau)^{8}}{\eta(\tau)^{4}}=\sum_{\substack{n=1 \\ n: \text { odd }}}^{\infty}\left(\sum_{d \mid n} d\right) q^{\frac{n}{2}}=q^{\frac{1}{2}}+4 q^{\frac{3}{2}}+6 q^{\frac{5}{2}}+8 q^{\frac{7}{2}}+\cdots
$$


will also appear in the formula. This is of weight 2 belonging to the principal congruence subgroup

$$
\Gamma(2)=\left\{\left(\begin{array}{ll}
a & b \\
c & d
\end{array}\right) \in \mathrm{SL}_{2}(\mathbb{Z}) \mid a \equiv d \equiv 1, b \equiv c \equiv 0 \bmod 2\right\},
$$

which is of index 2 in $\Gamma_{0}(2)$. (This is seen from the transformation formula of the eta function.)

Similarly, we define

$$
\begin{aligned}
E_{1}^{(3)}(\tau) & :=1+6 \sum_{n=1}^{\infty}\left(\sum_{d \mid n}\left(\frac{d}{3}\right)\right) q^{n}=1+6 q+6 q^{3}+6 q^{4}+\cdots \\
\Delta_{3}^{(3)}(\tau) & :=\frac{\eta(3 \tau)^{9}}{\eta(\tau)^{3}}=\sum_{n=1}^{\infty}\left(\sum_{d \mid n}\left(\frac{d}{3}\right)(n / d)^{2}\right) q^{n}=q+3 q^{2}+9 q^{3}+13 q^{4}+\cdots \\
j^{(3)}(\tau) & :=\frac{E_{1}^{(3)}(\tau)^{3}}{\Delta_{3}^{(3)}(\tau)}=\frac{1}{q}+15+54 q-76 q^{2}-\cdots
\end{aligned}
$$

where $\left(\frac{d}{3}\right)$ is the Legendre character. Both $E_{1}^{(3)}$ and $\Delta_{3}^{(3)}(\tau)$ are modular forms of weights 1 and 3 with character $\left(\frac{d}{3}\right)$ on the group $\Gamma_{0}(3)$, while the function $j^{(3)}(\tau)$ is a generator of the field of modular fuctions on $\Gamma_{0}(3)$. We also need

$$
\left(\Delta_{3}^{(3)}(\tau)\right)^{\frac{1}{3}}=\frac{\eta(3 \tau)^{3}}{\eta(\tau)}=\sum_{\substack{n=1 \\ n \neq 0(3)}}^{\infty}\left(\sum_{d \mid n}\left(\frac{d}{3}\right)\right) q^{n}=q^{\frac{1}{3}}+q^{\frac{4}{3}}+2 q^{\frac{7}{3}}+2 q^{\frac{13}{3}}+\cdots,
$$

which, as is shown similarly as in the case of $\sqrt{\Delta_{4}^{(2)}(\tau)}$ by the transformation formula of the eta function, is of weight 1 (with the same character) on the subgroup

$$
\Gamma_{0}^{0}(3)=\left\{\left(\begin{array}{ll}
a & b \\
c & d
\end{array}\right) \in \mathrm{SL}_{2}(\mathbb{Z}) \mid b \equiv c \equiv 0 \bmod 3\right\}
$$

of index 3 of $\Gamma_{0}(3)$. Finally, let

$$
\begin{aligned}
E_{2}^{(4)}(\tau) & :=\frac{1}{3}\left(4 E_{2}(4 \tau)-E_{2}(\tau)\right)=1+8 q+24 q^{2}+32 q^{3}+24 q^{4}+\cdots, \\
\Delta_{2}^{(4)}(\tau) & :=\frac{\eta(4 \tau)^{8}}{\eta(2 \tau)^{4}}=\sum_{\substack{n=1 \\
n: \text { odd }}}^{\infty}\left(\sum_{d \mid n} d\right) q^{n}=q+4 q^{3}+6 q^{5}+8 q^{7}+\cdots, \\
j^{(4)}(\tau) & :=\frac{E_{2}^{(4)}(\tau)}{\Delta_{2}^{(4)}(\tau)}=\frac{1}{q}+8+20 q-62 q^{3}+216 q^{5}-\cdots
\end{aligned}
$$


The functions $E_{2}^{(4)}(\tau)$ and $\Delta_{2}^{(4)}(\tau)$ are modular of weight 2 and 4 on $\Gamma_{0}(4)$ and $j^{(4)}(\tau)$ generates the field of modular functions on $\Gamma_{0}(4)$. Further we need

$$
\left(E_{2}^{(4)}(\tau)\right)^{\frac{1}{4}}=\theta_{3}(2 \tau)=\sum_{n \in \mathbb{Z}} q^{n^{2}}=1+2 q+2 q^{4}+2 q^{9}+\cdots
$$

which is of weight $1 / 2$ on $\Gamma_{0}(4)$ and

$$
\left(\Delta_{2}^{(4)}(\tau)\right)^{\frac{1}{4}}=\frac{\eta(4 \tau)^{2}}{\eta(2 \tau)}=\frac{1}{2} \theta_{2}(2 \tau)=\frac{1}{2} \sum_{n \in \mathbb{Z}} q^{\left(n+\frac{1}{2}\right)^{2}}=q^{\frac{1}{4}}+q^{\frac{9}{4}}+q^{\frac{25}{4}}+q^{\frac{49}{4}}+\cdots
$$

which is of weight $1 / 2$ on

$$
\Gamma_{0}^{0}(4)=\left\{\left(\begin{array}{ll}
a & b \\
c & d
\end{array}\right) \in \mathrm{SL}_{2}(\mathbb{Z}) \mid b \equiv c \equiv 0 \bmod 4\right\} .
$$

Here, $\theta_{3}$ and $\theta_{2}$ are Jacobi's theta functions (we do not use these notation later on). That the $\theta_{2}(2 \tau)$ belongs to $\Gamma_{0}^{0}(4)$ is seen from the transformation formula for theta functions or alternatively from the fact that $\theta_{2}(8 \tau)=\theta_{3}(2 \tau)-\theta_{3}(8 \tau)$ is a modular form of weight $1 / 2$ on $\Gamma_{0}(16)$ and that

$$
\Gamma_{0}^{0}(4)=\left(\begin{array}{ll}
4 & 0 \\
0 & 1
\end{array}\right) \Gamma_{0}(16)\left(\begin{array}{cc}
\frac{1}{4} & 0 \\
0 & 1
\end{array}\right)
$$

Recall the definition of the Gauss hypergeometric series $F={ }_{2} F_{1}$ :

$$
F(a, b, c ; x)=\sum_{n=0}^{\infty} \frac{(a)_{n}(b)_{n}}{(c)_{n}} \frac{x^{n}}{n !}
$$

where $(a)_{n}$ denotes $a(a+1) \cdots(a+n-1)$.

\section{Theorem 1.}

(i) When $k$ is an even integer congruent to 0 or 4 modulo 6 , the equation $(\#)_{k}$ has one dimensional solutions which are holomorphic modular forms of weight $k$ on $S L_{2}(\mathbb{Z})$, a generator of which is given by

$$
\begin{aligned}
& E_{4}(\tau)^{\frac{k}{4}} F\left(-\frac{k}{12},-\frac{k-4}{12},-\frac{k-5}{6} ; \frac{1728}{j(\tau)}\right) \\
& =\sum_{0 \leq i \leq k / 12} \frac{\left(-\frac{k}{12}\right)_{i}\left(-\frac{k-4}{12}\right)_{i}}{\left(-\frac{k-5}{6}\right)_{i} i !} 1728^{i} \Delta(\tau)^{i} E_{4}(\tau)^{\frac{k}{4}-3 i}=1+\mathrm{O}(q)
\end{aligned}
$$

if $k \equiv 0,4 \bmod 12$ and

$$
\begin{aligned}
& E_{4}(\tau)^{\frac{k-6}{4}} E_{6}(\tau) F\left(-\frac{k-6}{12},-\frac{k-10}{12},-\frac{k-5}{6} ; \frac{1728}{j(\tau)}\right) \\
& =E_{6}(\tau) \sum_{0 \leq i \leq(k-6) / 12} \frac{\left(-\frac{k-6}{12}\right)_{i}\left(-\frac{k-10}{12}\right)_{i}}{\left(-\frac{k-5}{6}\right)_{i} i !} 1728^{i} \Delta(\tau)^{i} E_{4}(\tau)^{\frac{k-6}{4}-3 i} \\
& =1+\mathrm{O}(q)
\end{aligned}
$$


if $k \equiv 6,10 \bmod 12$.

(ii) When $k$ is an even integer congruent to 2 modulo 6 , (\#) $)_{k}$ has two dimensional modular solutions: The function

$$
\begin{aligned}
& E_{2}^{(2)}(\tau)^{\frac{k}{2}} F\left(-\frac{k}{4},-\frac{k-2}{4},-\frac{k-5}{6} ; \frac{64}{j^{(2)}(\tau)}\right) \\
& =\sum_{0 \leq i \leq k / 4} \frac{\left(-\frac{k}{4}\right)_{i}\left(-\frac{k-2}{4}\right)_{i}}{\left(-\frac{k-5}{6}\right)_{i} i !} 64^{i} \Delta_{4}^{(2)}(\tau)^{i} E_{2}^{(2)}(\tau)^{\frac{k}{2}-2 i}=1+\mathrm{O}(q)
\end{aligned}
$$

which is a holomorphic modular form of weight $k$ on $\Gamma_{0}(2)$ generates a one dimensional subspace and the function

$$
\begin{aligned}
& \Delta_{4}^{(2)}(\tau)^{\frac{k+1}{6}} E_{2}^{(2)}(\tau)^{\frac{k-2}{6}} F\left(-\frac{k-2}{12},-\frac{k-8}{12}, \frac{k+7}{6} ; \frac{64}{j^{(2)}(\tau)}\right) \\
& =\sum_{0 \leq i \leq(k-2) / 12} \frac{\left(-\frac{k-2}{12}\right)_{i}\left(-\frac{k-8}{12}\right)_{i}}{\left(\frac{k+7}{6}\right)_{i} i !} 64^{i} \Delta_{4}^{(2)}(\tau)^{\frac{k+1}{6}+i} E_{2}^{(2)}(\tau)^{\frac{k-2}{6}-2 i} \\
& =q^{\frac{k+1}{6}}+\mathrm{O}\left(q^{\frac{k+7}{6}}\right)
\end{aligned}
$$

which is of weight $k$ on $\Gamma(2)$ constitutes the other generator.

(iii) When $k$ is an odd integer congruent to 1 or 3 modulo $6,(\#)_{k}$ has two dimensional modular solutions: The function

$$
\begin{aligned}
& E_{1}^{(3)}(\tau)^{k} F\left(-\frac{k}{3},-\frac{k-1}{3},-\frac{k-5}{6} ; \frac{27}{j^{(3)}(\tau)}\right) \\
& =\sum_{0 \leq i \leq k / 3} \frac{\left(-\frac{k}{3}\right)_{i}\left(-\frac{k-1}{3}\right)_{i}}{\left(-\frac{k-5}{6}\right)_{i} i !} 27^{i} \Delta_{3}^{(3)}(\tau)^{i} E_{1}^{(3)}(\tau)^{k-3 i}=1+O(q)
\end{aligned}
$$

which is a holomorphic modular form of weight $k$ on $\Gamma_{0}(3)$ generates a one dimensional subspace and the function

$$
\begin{aligned}
& \Delta_{3}^{(3)}(\tau)^{\frac{k+1}{6}} E_{1}^{(3)}(\tau)^{\frac{k-1}{2}} F\left(-\frac{k-1}{6},-\frac{k-3}{6}, \frac{k+7}{6} ; \frac{27}{j^{(3)}(\tau)}\right) \\
& =\sum_{0 \leq i \leq(k-1) / 6} \frac{\left(-\frac{k-1}{6}\right)_{i}\left(-\frac{k-3}{6}\right)_{i}}{\left(\frac{k+7}{6}\right)_{i} i !} 27^{i} \Delta_{3}^{(3)}(\tau)^{\frac{k+1}{6}+i} E_{1}^{(3)}(\tau)^{\frac{k-1}{2}-3 i} \\
& =q^{\frac{k+1}{6}}+\mathrm{O}\left(q^{\frac{k+7}{6}}\right)
\end{aligned}
$$

which is of weight $k$ on $\Gamma_{0}^{0}(3)$ constitutes the other generator.

(iv) When $k$ is half an integer congruent to $\frac{1}{2}$ modulo 3 , (\#) ${ }_{k}$ has two dimensional modular solutions: The function

$$
\begin{aligned}
& E_{2}^{(4)}(\tau)^{\frac{k}{2}} F\left(-\frac{2 k-1}{6},-\frac{k}{2},-\frac{k-5}{6} ; \frac{16}{j^{(4)}(\tau)}\right) \\
& =\sum_{0 \leq i \leq(2 k-1) / 6} \frac{\left(-\frac{2 k-1}{6}\right)_{i}\left(-\frac{k}{2}\right)_{i}}{\left(-\frac{k-5}{6}\right)_{i} i !} 16^{i} \Delta_{2}^{(4)}(\tau)^{i} E_{2}^{(4)}(\tau)^{\frac{k}{2}-i}=1+O(q)
\end{aligned}
$$


which is a holomorphic modular form of half-integral weight $k$ on $\Gamma_{0}(4)$ generates a one dimensional subspace and the function

$$
\begin{aligned}
& \Delta_{2}^{(4)}(\tau)^{\frac{k+1}{6}} E_{2}^{(4)}(\tau)^{\frac{2 k-1}{6}} F\left(-\frac{2 k-1}{6},-\frac{k-2}{6}, \frac{k+7}{6} ; \frac{16}{j^{(4)}(\tau)}\right) \\
& =\sum_{0 \leq i \leq(2 k-1) / 6} \frac{\left(-\frac{2 k-1}{6}\right)_{i}\left(-\frac{k-2}{6}\right)_{i}}{\left(\frac{k+7}{6}\right)_{i} i !} 16^{i} \Delta_{2}^{(4)}(\tau)^{\frac{k+1}{6}+i} E_{2}^{(4)}(\tau)^{\frac{2 k-1}{6}-i} \\
& =q^{\frac{k+1}{6}}+\mathrm{O}\left(q^{\frac{k+7}{6}}\right)
\end{aligned}
$$

which is of weight $k$ on $\Gamma_{0}^{0}(4)$ constitutes the other generator.

Remark. 1. The case (1) is contained in [1, Theorem 5].

2. We expect by numerical evidence no other modular solution to $(\#)_{k}$, at least when $k$ is an integer or half an integer (denominators of coefficients of power series solution in other cases seem not to be bounded). We however have no proof of this speculation.

Before giving a proof, we introduce the operator $\partial_{k}$ defined by

$$
\partial_{k}(f)(\tau)=f^{\prime}(\tau)-\frac{k}{12} E_{2}(\tau) f(\tau)
$$

By the quasimodular property of $E_{2}(\tau)$ which reads

$$
E_{2}\left(\frac{a \tau+b}{c \tau+d}\right)=(c \tau+d)^{2} E_{2}(\tau)+\frac{6}{\pi i} c(c \tau+d) \quad\left(\left(\begin{array}{ll}
a & b \\
c & d
\end{array}\right) \in \mathrm{SL}_{2}(\mathbb{Z})\right),
$$

we see that if $f$ is modular of weight $k$ on a subgroup of $\mathrm{SL}_{2}(\mathbb{Z})$, then $\partial_{k}(f)$ is modular of weight $k+2$ on the same group. If $f$ and $g$ have weights $k$ and $l$, the Leibniz rule

$$
\partial_{k+l}(f g)=\partial_{k}(f) g+f \partial_{l}(g)
$$

holds. We shall often drop the suffix of the operator $\partial_{k}$ since the weights of modular forms we shall be considering are clear in most cases. With this operator, the equation $(\#)_{k}$ can be written as

$$
\left(\#^{\prime}\right)_{k} \quad \partial_{k+2} \partial_{k}(f)(\tau)=\frac{k(k+2)}{144} E_{4}(\tau) f(\tau)
$$

(use $\left.E_{2}^{\prime}=\left(E_{2}^{2}-E_{4}\right) / 12\right)$.

Proof of Theorem 1. Given a specific modular form in terms of known forms, it is a straightforward task to check if it satisfies $(\#)_{k}$ or not. We give a proof of (2) to illustrate the calculation, the remaining cases being similar. Write $A=\Delta_{4}^{(2)}, B=$ $E_{2}^{(2)}$ to ease notation. We have

$$
\partial_{4}(A)=\frac{2}{3} A B, \partial_{2}(B)=32 A-\frac{1}{6} B^{2}
$$


and

$$
E_{4}=192 A+B^{2} .
$$

(To establish these kind of identities, it is enough to check that the first several Fourier coefficients coincide, since both sides of these equations are holomorphic modular forms of weight 6,4 and 4 on $\Gamma_{0}(2)$.) Using the first two we obtain

$$
\partial^{2}\left(A^{i} B^{\frac{k}{2}-2 i}\right)=a A^{i} B^{\frac{k}{2}-2 i+2}+b A^{i+1} B^{\frac{k}{2}-2 i}+c A^{i+2} B^{\frac{k}{2}-2 i-2}
$$

with

$a=\frac{(k-12 i)(k-12 i+2)}{144}, b=-\frac{8}{3}((k-4 i)(k-12 i-4)-8 i), c=256(k-4 i)(k-4 i-2)$.

Hence, for

$$
f=\sum_{0 \leq i \leq k / 4} c_{i} A^{i} B^{\frac{k}{2}-2 i} \quad \text { with } \quad c_{i}=64^{i} \frac{\left(-\frac{k}{4}\right)_{i}\left(-\frac{k-2}{4}\right)_{i}}{\left(-\frac{k-5}{6}\right)_{i} i !}
$$

we have

$$
\partial^{2}(f)-\frac{k(k+2)}{144} E_{4} f=\sum_{0 \leq i \leq k / 4} c_{i}^{\prime} A^{i+1} B^{\frac{k}{2}-2 i},
$$

where

$$
\begin{aligned}
c_{i}^{\prime} & =\frac{(k-12 i-12)(k-12 i-10)}{144} c_{i+1}-\frac{8}{3}((k-4 i)(k-12 i-4)-8 i) c_{i} \\
& +256(k-4 i+4)(k-4 i+2) c_{i-1}-\frac{k(k+2)}{144}\left(c_{i+1}+192 c_{i}\right) \\
& =c_{i} \times\left\{-\frac{1}{6} \frac{(k-12 i-12)(k-12 i-10)(k-4 i)(k-4 i-2)}{(k-6 i-5)(i+1)}\right. \\
& -\frac{8}{3}((k-4 i)(k-12 i-4)-8 i)-\frac{64}{6}(k-6 i+1) i \\
& \left.-\frac{k(k+2)}{144}\left(-24 \frac{(k-4 i)(k-4 i-2)}{(k-6 i-5)(i+1)}+192\right)\right\},
\end{aligned}
$$

which turns out to be identically 0 .

Next theorem describes a solution in the case of $k \equiv 5 \bmod 6$. Here we come across a different phenomenon: the equation $(\#)_{k}$ has quasimodular solutions of weight $k+1$ rather than $k$. Recall that an element of degree $k$ in the graded ring $\mathbb{C}\left[E_{2}(\tau), E_{4}(\tau), E_{6}(\tau)\right]$, where the generators $E_{2}, E_{4}, E_{6}$ have degrees 2,4 , and 6 , is referred to as a quasimodular form of weight $k$ (on $\mathrm{SL}_{2}(\mathbb{Z})$ ). Define a sequence of polynomials $P_{n}(x)(n=0,1,2, \ldots)$ by

$$
P_{0}(x)=1, P_{1}(x)=x, \quad P_{n+1}(x)=x P_{n}(x)+\lambda_{n} P_{n-1}(x)(n=1,2, \ldots)
$$


where

$$
\lambda_{n}=12 \frac{(6 n+1)(6 n+5)}{n(n+1)} .
$$

First few examples are

$$
P_{2}(x)=x^{2}+462, P_{3}(x)=x^{3}+904 x, P_{4}(x)=x^{4}+1341 x^{2}+201894, \ldots
$$

Clearly $P_{n}(x)$ is even or odd polynomial according as $n$ is even or odd. We also define a series of "companion" polynomials $Q_{n}(x)$ by the same recursion (with different initial values):

$$
Q_{0}(x)=0, Q_{1}(x)=1, \quad Q_{n+1}(x)=x Q_{n}(x)+\lambda_{n} Q_{n-1}(x)(n=1,2, \ldots),
$$

a few examples being

$$
Q_{2}(x)=x, Q_{3}(x)=x^{2}+442, Q_{4}(x)=x^{3}+879 x, \ldots
$$

The $Q_{n}(x)$ has opposite parity: It is even if $n$ is odd and odd if $n$ is even.

Theorem 2. Let $k=6 n+5(n=0,1,2, \ldots)$. The following quasimodular form of weight $k+1$ on $S L_{2}(\mathbb{Z})$ is a solution of $(\#)_{k}$ :

$$
\sqrt{\Delta(\tau)}^{n} P_{n}\left(\frac{E_{6}(\tau)}{\sqrt{\Delta(\tau)}}\right) \frac{E_{4}^{\prime}(\tau)}{240}-\sqrt{\Delta(\tau)}^{n+1} Q_{n}\left(\frac{E_{6}(\tau)}{\sqrt{\Delta(\tau)}}\right) .
$$

Remark. Because of the parities of $P_{n}(x)$ and $Q_{n}(x)$, the appearance of $\sqrt{\Delta(\tau)}$ in the formula is in fact superficial, i.e., it cancels out and the formula gives an element in $\mathbb{Q}\left[E_{2}(\tau), E_{4}(\tau), E_{6}(\tau)\right]$ (note $\left.E_{4}^{\prime}(\tau)=\left(E_{2}(\tau) E_{4}(\tau)-E_{6}(\tau)\right) / 3\right)$.

Since the polynomials $P_{n}(x)$ and $Q_{n}(x)$ are not hypergeometric polynomials, it is not at all straightforward to show the expression in the theorem satisfies $(\#)_{k}$. We shall give a proof of the theorem in the next section where an inductive structure of solutions of $(\#)_{k}$ with varying $k$ is discussed.

A few more words about the polynomials $P_{n}(x)$ and $Q_{n}(x)$ : If we replace the constant $\lambda_{n}=12(6+1 / n)(6-1 /(n+1))$ in the recursion of $P_{n}$ and $Q_{n}$ by $12(6+$ $\left.(-1)^{n} /(n-1)\right)\left(6+(-1)^{n} / n\right)$, (even part of) the resulting polynomials $P_{n}(x)$ give "Atkin's orthogonal polynomials" (see [1, Sections 4 and 5]). In our case, the polynomials $P_{n}(x)$ and $Q_{n}(x)$ are connected to the convergents of the continued fraction expansion of

$$
\frac{1}{240} \frac{E_{4}^{\prime}}{E_{6}}=\frac{1}{j} \frac{F\left(\frac{13}{12}, \frac{17}{12}, 2 ; \frac{1728}{j}\right)}{F\left(\frac{1}{12}, \frac{5}{12}, 1 ; \frac{1728}{j}\right)}=\frac{1}{j}+\frac{1266}{j^{2}}+\frac{1806960}{j^{3}}+\cdots,
$$

in a similar manner as in Atkin's case where the function involved was

$$
\frac{E_{2} E_{4}}{E_{6} j}=\frac{1}{j} \frac{F\left(\frac{13}{12}, \frac{5}{12}, 1 ; \frac{1728}{j}\right)}{F\left(\frac{1}{12}, \frac{5}{12}, 1 ; \frac{1728}{j}\right)}=\frac{1}{j}+\frac{720}{j^{2}}+\frac{911520}{j^{3}}+\cdots .
$$

It should be possible, by an analogous method as in [1], to establish properties like a closed formula or a differential equation of our $P_{n}$ and $Q_{n}$. We however do not pursue this here. 


\section{§3. INDUCTIVE STRUCTURE OF SOLUTIONS}

For modular forms $f(\tau)$ and $g(\tau)$ of weights $k$ and $l$, define a modular form $[f(\tau), g(\tau)]$ of weight $k+l+2$ by

$$
[f(\tau), g(\tau)]=k f(\tau) g^{\prime}(\tau)-l f^{\prime}(\tau) g(\tau)
$$

("Rankin-Cohen bracket" of degree 1). The right-hand side may also be written as

$$
k f(\tau) \partial_{l}(g(\tau))-l \partial_{k}(f(\tau)) g(\tau) .
$$

Lemma. Suppose $f=F_{k}(\tau)$ satisfies $\left(\#^{\prime}\right)_{k}$. Then we have

$$
\partial_{k+6}\left(\left[F_{k}(\tau), E_{4}(\tau)\right]\right)=\frac{k-4}{18}\left[F_{k}(\tau), E_{6}(\tau)\right]
$$

and

$$
\partial_{k+8}\left(\left[F_{k}(\tau), E_{6}(\tau)\right]\right)=\frac{k-6}{8} E_{4}(\tau)\left[F_{k}(\tau), E_{4}(\tau)\right] .
$$

Here, in the Rankin-Cohen brackets, the function $F_{k}(\tau)$ is regarded as being of weight $k$.

Proof. Using $\partial\left(E_{4}\right)=-E_{6} / 3$ and $\partial\left(E_{6}\right)=-E_{4}^{2} / 2$, we have

$$
\begin{aligned}
\partial\left(\left[F_{k}, E_{4}\right]\right) & =\partial\left(-\frac{k}{3} F_{k} E_{6}-4 \partial\left(F_{k}\right) E_{4}\right) \\
& =-\frac{k}{3} \partial\left(F_{k}\right) E_{6}+\frac{k}{6} F_{k} E_{4}^{2}-4\left(\frac{k(k+2)}{144} E_{4} F_{k}\right) E_{4}+\frac{4}{3} \partial\left(F_{k}\right) E_{6} \\
& =-\frac{k(k-4)}{36} F_{k} E_{4}^{2}-\frac{k-4}{3} \partial\left(F_{k}\right) E_{6} \\
& =\frac{k-4}{18}\left[F_{k}, E_{6}\right] .
\end{aligned}
$$

The other identity is shown similarly.

\section{Proposition 1.}

(i) If $F_{k}(\tau)$ is a solution of $(\#)_{k}$, then $\left[F_{k}(\tau), E_{4}(\tau)\right] / \Delta(\tau)$ is a solution of $(\#)_{k-6}$.

(ii) Assume $k \neq 0,4,5$. Given a solution $F_{k}(\tau)$ of $(\#)_{k}$, put

$$
F_{k-6}(\tau)=\frac{k-5}{288 k(k-4)} \frac{\left[F_{k}(\tau), E_{4}(\tau)\right]}{\Delta(\tau)}
$$

and define $F_{k+6 i}(\tau)(i=1,2,3, \ldots)$ successively by the recursion

$F_{k+6 i+6}(\tau)=E_{6}(\tau) F_{k+6 i}(\tau)+\mu_{i}^{(k)} \Delta(\tau) F_{k+6 i-6}(\tau) \quad(i=0,1,2, \ldots)$

where

$$
\mu_{i}^{(k)}=432 \frac{(k+6 i)(k+6 i-4)}{(k+6 i+1)(k+6 i-5)} .
$$

Then $F_{k+6 i}(\tau)$ is a solution of $(\#)_{k+6 i}$ for every $i$. 
Proof. Noting $\partial(\Delta(\tau))=0$ (since $\Delta^{\prime}=E_{2} \Delta$ ), the assertion (1) is readily shown as a direct consequence of Lemma. In fact, we have

$$
\partial^{2}\left(\frac{\left[F_{k}, E_{4}\right]}{\Delta}\right)=\frac{\partial^{2}\left(\left[f_{k}, E_{4}\right]\right)}{\Delta}=\frac{k-4}{18} \frac{\partial\left(\left[F_{k}, E_{6}\right]\right)}{\Delta}=\frac{(k-6)(k-4)}{144} E_{4} \frac{\left[F_{k}, E_{4}\right]}{\Delta},
$$

which shows that the function $\left[F_{k}, E_{4}\right] / \Delta$ satisfies the equivalent form $\left(\#^{\prime}\right)_{k-6}$ of $(\#)_{k-6}$.

For (2), we first show the following.

Lemma. If $G_{k}$ and $G_{k-6}$ are solutions of $(\#)_{k}$ and $(\#)_{k-6}$ respectively, then the function $G_{k+6}:=E_{6} G_{k}+\Delta G_{k-6}$ is a solution of $(\#)_{k+6}$ if and only if the relation

$$
\left[G_{k}, E_{4}\right]=\frac{2}{3}(k+1) \Delta G_{k-6}
$$

holds.

Proof. Using

$$
\begin{aligned}
& \partial\left(E_{6} G_{k}\right)=-\frac{E_{4}^{2}}{2} G_{k}+E_{6} \partial\left(G_{k}\right)=-\frac{1}{6}\left[G_{k}, E_{6}\right]-\frac{k+6}{12} E_{4}^{2} G_{k}, \\
& \partial\left(E_{4}^{2} G_{k}\right)=-\frac{2}{3} E_{4} E_{6} G_{k}+E_{4}^{2} \partial\left(G_{k}\right)=-\frac{1}{4}\left[G_{k}, E_{4}\right]-\frac{k+4}{12} E_{6} G_{k},
\end{aligned}
$$

and the preceding lemma, we have

$$
\begin{aligned}
\partial^{2}\left(G_{k+6}\right) & =\partial^{2}\left(E_{6} G_{k}\right)+\Delta \partial^{2}\left(G_{k-6}\right) \\
& =\partial\left(-\frac{1}{6}\left[G_{k}, E_{6}\right]-\frac{k+6}{12} E_{4}^{2} G_{k}\right)+\Delta \partial^{2}\left(G_{k-6}\right) \\
& =-\frac{1}{6} \frac{k-6}{8} E_{4}\left[G_{k}, E_{4}\right]-\frac{k+6}{12}\left(-\frac{1}{4} E_{4}\left[G_{k}, E_{4}\right]-\frac{k+8}{12} E_{4} E_{6} G_{k}\right) \\
& +\Delta \frac{(k-6)(k-4)}{144} E_{4} G_{f-6} \\
& =\frac{1}{4} E_{4}\left[G_{k}, E_{4}\right]+\frac{(k+6)(k+8)}{144} E_{4} E_{6} G_{k}+\frac{(k-6)(k-4)}{144} E_{4} \Delta G_{k-6} .
\end{aligned}
$$

Hence we obtain

$$
\partial^{2}\left(G_{k+6}\right)-\frac{(k+6)(k+8)}{144} E_{4} G_{k+6}=\frac{E_{4}}{4}\left(\left[G_{k}, E_{4}\right]-\frac{2}{3}(k+1) \Delta G_{k-6}\right) .
$$

The lemma follows from this.

We return to the proof of (2) of Proposition 1. By (1), the function $\mu_{0} F_{k-6}$ is a solution of $(\#)_{k-6}$ and it is so defined that the relation

$$
\left[F_{k}, E_{4}\right]=\frac{2}{3}(k+1) \Delta\left(\mu_{0} F_{k-6}\right)
$$


holds. By the lemma we have just shown, we conclude that $F_{k+6}=E_{6} F_{k}+\mu_{0} \Delta F_{k-6}$ is a solution of $(\#)_{k+6}$. Moreover, the pair $F_{k+6}$ and $\mu_{1} F_{k}$ satisfy

$$
\left[F_{k+6}, E_{4}\right]=\frac{2}{3}(k+7) \Delta\left(\mu_{1} F_{k}\right)
$$

as the following calculation shows;

$$
\begin{aligned}
{\left[F_{k+6}, E_{4}\right] } & =\left[E_{6} F_{k}+\mu_{0} \Delta F_{k-6}, E_{4}\right]=\left[E_{6} F_{k}+\frac{3}{2(k+1)}\left[F_{k}, E_{4}\right], E_{4}\right] \\
& =(k+6) E_{6} F_{k} \partial\left(E_{4}\right)-4 \partial\left(E_{6} F_{k}\right) E_{4} \\
& +\frac{3}{2(k+1)}\left((k+6)\left[F_{k}, E_{4}\right] \partial\left(E_{4}\right)-4 \partial\left(\left[F_{k}, E_{4}\right]\right) E_{4}\right) \\
& =\frac{k+6}{3} E_{6}^{2} F_{k}+2 E_{4}^{3} F_{k}-4 E_{4} E_{6} \partial\left(F_{k}\right) \\
& -\frac{k+6}{2(k+1)} E_{6}\left(-\frac{k}{3} E_{6} F_{k}-4 E_{4} \partial\left(F_{k}\right)\right) \\
& -\frac{6}{k+1} E_{4} \frac{k-4}{18}\left(-\frac{k}{2} E_{4}^{2} F_{k}-6 E_{6} \partial\left(F_{k}\right)\right) \\
& =\frac{(k+2)(k+6)}{6(k+1)}\left(E_{4}^{3}-E_{6}^{2}\right) F_{k}=\frac{2}{3}(k+7) \mu_{1} \Delta F_{k} .
\end{aligned}
$$

Thus, applying the lemma again to conclude $F_{k+12}$ is a solution of $(\#)_{k+12}$. By replacing $k$ with $k+6$ in the above calculation, we see this procedure continues inductively and the proposition is proved.

Proof of Theorem 2. For $k=6 n+5(n=0,1,2, \ldots)$, denote by $F_{k}(\tau)$ the form in the theorem,

$$
F_{k}(\tau)=\sqrt{\Delta(\tau)}^{n} P_{n}\left(\frac{E_{6}(\tau)}{\sqrt{\Delta(\tau)}}\right) \frac{E_{4}^{\prime}(\tau)}{240}-\sqrt{\Delta(\tau)}^{n+1} Q_{n}\left(\frac{E_{6}(\tau)}{\sqrt{\Delta(\tau)}}\right) .
$$

We see directly that $F_{11}=E_{6} E_{4}^{\prime} / 240-\Delta$ is a solution of $(\#)_{11}$ and that

$$
F_{5}=\frac{E_{4}^{\prime}}{240}=\frac{11-6}{288 \cdot 11 \cdot(11-4)} \frac{\left[F_{11}, E_{4}\right]}{\Delta} .
$$

By Proposition 1 (2), it is then enough to show that the $F_{k}$ 's satisfy the recursion in Proposition 1 (2). Alternatively, we may start with checking that $F_{5}=E_{4}^{\prime} / 240$ satisfies $(\#)_{5}$ and calculate $\left[F_{5}, E_{4}\right]=-4 \Delta$. By this, if we put $F_{-1}=1$ and $\mu_{0}^{(5)}=-1$, then the initial condition of Proposition 1 (2) is satisfied and also we have $F_{11}=E_{6} F_{5}+\mu_{0}^{(5)} \Delta F_{-1}$, so again the task is that the $F_{k}$ 's satisfy the recursion in Proposition 1 (2). Noticing the constant $\mu_{i}^{(k)}$ in Proposition 1 is equal to

$$
432 \frac{(6 n+5+6 i)(6 n+5+6 i-4)}{(6 n+5+6 i+1)(6 n+5+6 i-5)}=12 \frac{(6 n+6 i+5)(6 n+6 i+1)}{(n+i+1)(n+i)}=\lambda_{n+i},
$$


we have, by the recursion of $P_{n}$ and $Q_{n}$,

$$
\begin{aligned}
& E_{6} F_{k+6 i}+\mu_{i}^{(k)} \Delta F_{k+6 i-6} \\
& =E_{6}\left(\sqrt{\Delta}^{n+i} P_{n+i}\left(\frac{E_{6}}{\sqrt{\Delta}}\right) \frac{E_{4}^{\prime}}{240}-\sqrt{\Delta}^{n+i+1} Q_{n+i}\left(\frac{E_{6}}{\sqrt{\Delta}}\right)\right) \\
& +\lambda_{n+i} \Delta\left(\sqrt{\Delta}^{n+i-1} P_{n+i-1}\left(\frac{E_{6}}{\sqrt{\Delta}}\right) \frac{E_{4}^{\prime}}{240}-\sqrt{\Delta}^{n+i} Q_{n+i-1}\left(\frac{E_{6}}{\sqrt{\Delta}}\right)\right) \\
& =\sqrt{\Delta}^{n+i+1}\left(\frac{E_{6}}{\sqrt{\Delta}} P_{n+i}\left(\frac{E_{6}}{\sqrt{\Delta}}\right)+\lambda_{n+i} P_{n+i-1}\left(\frac{E_{6}}{\sqrt{\Delta}}\right)\right) \frac{E_{4}^{\prime}}{240} \\
& -\sqrt{\Delta}^{n+i+2}\left(\frac{E_{6}}{\sqrt{\Delta}} Q_{n+i}\left(\frac{E_{6}}{\sqrt{\Delta}}\right)+\lambda_{n+i} Q_{n+i-1}\left(\frac{E_{6}}{\sqrt{\Delta}}\right)\right) \\
& =\sqrt{\Delta}^{n+i+1} P_{n+i+1}\left(\frac{E_{6}}{\sqrt{\Delta}}\right) \frac{E_{4}^{\prime}}{240}-\sqrt{\Delta}^{n+i+2} Q_{n+i+1}\left(\frac{E_{6}}{\sqrt{\Delta}}\right) \\
& =F_{k+6 i+6 .} .
\end{aligned}
$$

We therefore conclude that $F_{k+6 i}$ is a solution of $(\#)_{k+6 i}$ for every $i$.

Remark. The recursion in Proposition 1 is also satisfied by the modular solutions given in Theorem 1 . In that case, the recursion reduces to relations of hypergeometric series but we should point out that those relations are not in general the relations referred to as the contiguous relations of Gauss. For instance, the first solution in (ii) of Theorem 1 (the case of $k \equiv 2 \bmod 6$ ) gives an identity of hypergeometric series of the form (which is true for any $k$ )

$$
\begin{aligned}
& x^{3} F\left(-\frac{k+6}{4},-\frac{k+4}{4},-\frac{k+1}{6} ; \frac{64}{x}\right) \\
& =\left(x^{3}-576 x^{2}\right) F\left(-\frac{k}{4},-\frac{k-2}{4},-\frac{k-5}{6} ; \frac{64}{x}\right) \\
& +432 \frac{k(k-4)}{(k-5)(k+1)}\left(x^{2}-128 x+4096\right) F\left(-\frac{k-6}{4},-\frac{k-8}{4},-\frac{k-11}{6} ; \frac{64}{x}\right),
\end{aligned}
$$

where $x=j^{(2)}=B^{2} / A$ (the polynomials on the right-hand side come from the identities

$$
E_{6}=B^{3}-576 A B, \quad \Delta=A B^{4}-128 A^{2} B^{2}+4096 A^{3},
$$

notation being as in the proof of Theorem 1). Instead of checking this sort of hypergeometric identities case by case in order to show the recursion for solutions in Theorem 1, we argue as follows. By the form of the recursion in Proposition 1 (ii), if $F_{k}$ has $q$-expansion of the form $1+\mathrm{O}(q)$ (with only integral powers of $q$ ), so does every $F_{k+6 i}$ (use $E_{6}=1+\mathrm{O}(q)$ and $\Delta=q+\mathrm{O}\left(q^{2}\right)$ ). Hence, starting with a solution in Theorem 1 of the form $1+\mathrm{O}(q)$ with $0<k \leq 6$ or $k=10$, we conclude by the uniqueness of solution of this form that the recursively determined solutions must coincide the ones in Theorem 1. The case of other solutions with $q^{(k+1) / 6}+\mathrm{O}\left(q^{(k+7) / 6}\right)$ is similar. Hence, the above hypergeometric identity or the other corresponding ones may be regarded as consequences of Theorem 1. 
Proposition 1 shows that if we find any solution of $(\#)_{k}$, we can construct solutions of $(\#)_{k}$ for larger $k$ in the same residue class modulo 6. Conversely, any solution "comes from" lower ones in this way. In fact, suppose $F_{k}$ is a solution of $(\#)_{k}$. Applying Proposition 1 (i) twice we see not only the function $\left[F_{k}, E_{4}\right] / \Delta$ is a solution of $(\#)_{k-6}$ but also the function $\left[\left[F_{k}, E_{4}\right] / \Delta, E_{4}\right] / \Delta$ is a solution of $(\#)_{k-12}$. Put

$$
F_{k-6}=\frac{k-5}{288 k(k-4)} \frac{\left[F_{k}, E_{4}\right]}{\Delta}
$$

and

$$
F_{k-12}=\frac{k-11}{288(k-6)(k-10)} \frac{\left[F_{k-6}, E_{4}\right]}{\Delta} .
$$

With these we have

$$
F_{k}=E_{6} F_{k-6}+\mu_{0}^{(k-6)} F_{k-12} .
$$

This tells us that the $F_{k}$ is obtained from $F_{k-6}$ as in Proposition 1 (ii). Taking account of this consideration and the fact that if $F_{k}$ is a holomorphic modular form then so is $\left[F_{k}, E_{4}\right] / \Delta$, in order to find a modular solution of $(\#)_{k}$, we may restrict ourselves to find one in the range $0 \leq k<6$. (Since $\left[1, E_{4}\right]=\left[E_{4}, E_{4}\right]=0$, Proposition 1 (ii) is valid for $k=0$ or 4 if we take $F_{0}=1$ or $F_{4}=E_{4}$ with $F_{-6}=0$ or $F_{-2}=0$.)

Remarkably enough, in a completely different context (deformation of singularities etc.) the equation $(\#)_{k}$ (in its yet another equivalent form different from $\left.\left(\#^{\prime}\right)_{k}\right)$ for integral or half-integral $k$ in this range was considered in Ikuo Satake $[2,3]$. There, the solutions are expressed as theta functions associated with positive definite lattices, a table of which is given below:

\begin{tabular}{c|c|c|c|c|c|c|c|c|c|c|c|c}
$k$ & 0 & $\frac{1}{2}$ & 1 & $\frac{3}{2}$ & 2 & $\frac{5}{2}$ & 3 & $\frac{7}{2}$ & 4 & $\frac{9}{2}$ & 5 & $\frac{11}{2}$ \\
\hline$F_{k}$ & 1 & $\Theta_{A_{1}}$ & $\Theta_{A_{2}}$ & - & $\Theta_{D_{4}}$ & - & $\Theta_{E_{6}}$ & $\Theta_{E_{7}}$ & $\Theta_{E_{8}}$ & - & - & - \\
& & $\Theta_{A_{1}^{*}}$ & $\Theta_{A_{2}^{*}}$ & & $\Theta_{D_{4}^{*}}$ & & $\Theta_{E_{6}^{*}}$ & $\Theta_{E_{7}^{*}}$ & & & &
\end{tabular}

Here, $\Theta_{L}$ is the theta function of a lattice $L$ and $A_{n}, D_{n}, E_{n}$ denote root lattices while $A_{n}^{*}, D_{n}^{*}, E_{n}^{*}$ are their duals. In Satake's investigation, the existence of modular solution in that range (e.g., we have a modular solution for $k=1 / 2$ but not for $k=3 / 2$ ) corresponds exactly to the existence of certain affine root system. It seems to be an interesting question if the existence or non-existence of modular solution of $(\#)_{k}$ is explained by any number theoretical reason. Also, the meaning of the quasimodular solution $E_{4}^{\prime}$ (for $k=5$ ) and the ones for higher weights should be clarified.

\section{$\S 4$. Positiveness of Fourier coefficients}

As remarked in the previous section, some solutions of $(\#)_{k}$ for small $k$ are theta series of positive definite lattices and hence have positive Fourier coefficients. For general $k$, we prove the following. 
Theorem 3. All the solutions given in Theorem 1 with q-expansion of the form $q^{(k+1) / 6}+\mathrm{O}\left(q^{(k+7) / 6}\right)$ have positive Fourier coefficients.

Proof. First we prove the case $k \equiv 2 \bmod 6$. The solution in question is

$$
\sum_{0 \leq i \leq(k-2) / 12} \frac{\left(-\frac{k-2}{12}\right)_{i}\left(-\frac{k-8}{12}\right)_{i}}{\left(\frac{k+7}{6}\right)_{i} i !} 64^{i} \Delta_{4}^{(2)}(\tau)^{\frac{k+1}{6}+i} E_{2}^{(2)}(\tau)^{\frac{k-2}{6}-2 i}
$$

Since the product $(-(k-2) / 12)_{i}(-(k-8) / 12)_{i}$ in the numerator in the coefficient is always positive for $0 \leq i \leq(k-2) / 12$ and the forms

$$
\begin{aligned}
& E_{2}^{(2)}(\tau)=1+24 \sum_{n=1}^{\infty}\left(\sum_{\substack{d \mid n \\
d: \text { odd }}} d\right) q^{n} \\
& \Delta_{4}^{(2)}(\tau)=\sum_{n=1}^{\infty}\left(\sum_{\substack{d \mid n \\
d: \text { odd }}}(n / d)^{3}\right) q^{n}
\end{aligned}
$$

have positive coefficients, the solution above clearly has positive Fourier coefficients. The case when $k \equiv 1,3 \bmod 6$ is treated similarly: The product $(-(k-1) / 6)_{i}(-(k-$ $3) / 6)_{i}$ in the formula is positive and the forms

$$
E_{1}^{(3)}(\tau)=1+6 \sum_{n=1}^{\infty}\left(\sum_{d \mid n}\left(\frac{d}{3}\right)\right) q^{n}
$$

and

$$
\Delta_{3}^{(3)}(\tau)=\frac{\eta(3 \tau)^{9}}{\eta(\tau)^{3}}=\sum_{n=1}^{\infty}\left(\sum_{d \mid n}\left(\frac{d}{3}\right)(n / d)^{2}\right) q^{n}
$$

have non-negative and positive Fourier coefficients respectively, as shown below. Notice that the coefficients are multiplicative and hence checking when $n$ is a power of prime is enough. If $n=p^{e}$ is a power of prime, we have

$$
1+\left(\frac{p}{3}\right)+\left(\frac{p^{2}}{3}\right)+\cdots+\left(\frac{p^{e}}{3}\right)= \begin{cases}1, & p=3 \\ e+1, & \left(\frac{p}{3}\right)=1 \\ 1, & \left(\frac{p}{3}\right)=-1 \text { and } e=\text { even } \\ 0, & \left(\frac{p}{3}\right)=-1 \text { and } e=\text { odd }\end{cases}
$$

and

$$
p^{2 e}+\left(\frac{p}{3}\right) p^{2 e-2}+\left(\frac{p^{2}}{3}\right) p^{2 e-4}+\cdots+\left(\frac{p^{e}}{3}\right)= \begin{cases}p^{2 e}, & p=3, \\ \frac{p^{2 e+2}-1}{p^{2}-1}, & \left(\frac{p}{3}\right)=1, \\ \frac{p^{2 e+2}+1}{p^{2}+1}, & \left(\frac{p}{3}\right)=-1 \text { and } e=\text { even } \\ \frac{p^{2 e+2}-1}{p^{2}+1}, & \left(\frac{p}{3}\right)=-1 \text { and } e=\text { odd. }\end{cases}
$$


From this we conclude that $\sum_{d \mid n}\left(\frac{d}{3}\right) \geq 0$ and $\sum_{d \mid n}\left(\frac{d}{3}\right)(n / d)^{2}>0$.

When $k$ is of the form $(6 n+1) / 2$, the product $(-(2 k-1) / 6)_{i}(-(k-2) / 6)_{i}$ needs not always be positive and so we need some extra work. Put

$$
a_{i}=\frac{\left(-\frac{2 k-1}{6}\right)_{i}\left(-\frac{k-2}{6}\right)_{i}}{\left(\frac{k+7}{6}\right)_{i} i !}=\frac{(-n)_{i}\left(-\frac{2 n-1}{4}\right)_{i}}{\left(\frac{2 n+5}{4}\right)_{i} i !} .
$$

We have to show that the form

$$
\Delta_{2}^{(4)}(\tau)^{\frac{2 n+1}{4}} \sum_{i=0}^{n} a_{i}\left(16 \Delta_{2}^{(4)}(\tau)\right)^{i} E_{2}^{(4)}(\tau)^{n-i}
$$

has positive Fourier coefficients. Put $A=\Delta_{2}^{(4)}(\tau)$ and $B=E_{2}^{(4)}(\tau)$ as in the proof of Theorem 1. Note that the number $a_{i}$ is positive for $i<(2 n+3) / 4$ and beyond this it alternates the sign. Suppose now $a_{i}$ is positive and $a_{i+1}$ negative (or zero). Since $\Delta_{2}^{(4)}=\sum_{n \geq 1, \text { odd }}\left(\sum_{d \mid n} d\right) q^{n}$ has positive coefficients, our proof is complete if we show that the Fourier coefficients of

$$
\begin{aligned}
& a_{i}(16 A)^{i} B^{n-i}+a_{i+1}(16 A)^{i+1} B^{n-i-1} \\
& =a_{i}(16 A)^{i} B^{n-i-1}\left(B+16 \frac{(-n+i)\left(-\frac{2 n-1}{4}+i\right)}{\left(\frac{2 n+5}{4}+i\right)(i+1)} A\right)
\end{aligned}
$$

are positive. For this we prove the following lemma.

Lemma. For $\alpha$ with $0 \leq \alpha<8$, the Fourier coefficients in $E_{2}^{(4)}(\tau)-\alpha \Delta_{2}^{(4)}(\tau)$ are positive.

Proof. Denote the sum $\sum_{d \mid n} d$ by $\sigma(n)$. By

$$
E_{2}^{(4)}(\tau)=\frac{1}{3}\left(4 E_{2}(4 \tau)-E_{2}(\tau)\right)=1+8 \sum_{n=1}^{\infty} \sigma(n) q^{n}-32 \sum_{n=1}^{\infty} \sigma(n) q^{4 n}
$$

and

$$
\Delta_{2}^{(4)}(\tau)=\sum_{n \geq 1, \mathrm{odd}} \sigma(n) q^{n}
$$

we have

$$
\begin{aligned}
& E_{2}^{(4)}(\tau)-\alpha \Delta_{2}^{(4)}(\tau) \\
& =1+8 \sum_{n \equiv 2 \bmod 4} \sigma(n) q^{n}+8 \sum_{n=1}^{\infty}(\sigma(4 n)-4 \sigma(n)) q^{4 n}+\sum_{n \geq 1, \text { odd }}(8-\alpha) \sigma(n) q^{n} .
\end{aligned}
$$

To see the coefficient in the middle sum is positive, write $n=2^{e} m, m$ : odd. Then we have

$$
\begin{aligned}
\sigma(4 n)-4 \sigma(n) & =\sigma\left(2^{e+2} m\right)-4 \sigma\left(2^{e} m\right)=\left(\sigma\left(2^{e+2}\right)-4 \sigma\left(2^{e}\right)\right) \sigma(m) \\
& =\left(2^{e+3}-1-4\left(2^{e+1}-1\right)\right) \sigma(m)=3 \sigma(m)>0 .
\end{aligned}
$$


Thus we have proved the lemma.

In view of the lemma, the proof of Theorem 3 is complete if the inequality

$$
0 \leq-16 \frac{(-n+i)\left(-\frac{2 n-1}{4}+i\right)}{\left(\frac{2 n+5}{4}+i\right)(i+1)}<8 \text { for } \quad \frac{2 n+3}{4}<i \leq n
$$

is shown to hold, which is readily seen.

\section{§5. A characterization of the EQUation $(\#)_{k}$}

Consider the differential equation in the upper half-plane of the form

$$
f^{\prime \prime}(\tau)+A(\tau) f^{\prime}(\tau)+B(\tau) f(\tau)=0
$$

where $A(\tau)$ and $B(\tau)$ are assumed to be holomorphic in $\mathfrak{H}$ and bounded when $\Im(\tau) \rightarrow \infty$. Fix a non-negative integer $k$ and we further assume:

If $f(\tau)$ is a solution of $(1)$, then $(c \tau+d)^{-k} f\left(\frac{a \tau+b}{c \tau+d}\right)$ is also a solution of (1) for all $\left(\begin{array}{ll}a & b \\ c & d\end{array}\right) \in \mathrm{SL}_{2}(\mathbb{Z})$.

Proposition 2. The differential equation $(\#)_{k}$ is essentially the unique equation which satisfies the above conditions.

Proof. Put $g(\tau)=(c \tau+d)^{-k} f\left(\frac{a \tau+b}{c \tau+d}\right)$. By an elementary calculation, we have from the assumption

$$
\begin{aligned}
0 & =(c \tau+d)^{k+4}\left(g^{\prime \prime}(\tau)+A(\tau) g^{\prime}(\tau)+B(\tau) g(\tau)\right) \\
& =f^{\prime \prime}\left(\frac{a \tau+b}{c \tau+d}\right)+\left((c \tau+d)^{2} A(\tau)-\frac{k+1}{\pi i} c(c \tau+d)\right) f^{\prime}\left(\frac{a \tau+b}{c \tau+d}\right) \\
& +\left((c \tau+d)^{4} B(\tau)-\frac{k}{2 \pi i} c(c \tau+d)^{3} A(\tau)+\frac{k(k+1)}{(2 \pi i)^{2}} c^{2}(c \tau+d)^{2}\right) f\left(\frac{a \tau+b}{c \tau+d}\right) .
\end{aligned}
$$

Comparing this with

$$
f^{\prime \prime}\left(\frac{a \tau+b}{c \tau+d}\right)+A\left(\frac{a \tau+b}{c \tau+d}\right) f^{\prime}\left(\frac{a \tau+b}{c \tau+d}\right)+B\left(\frac{a \tau+b}{c \tau+d}\right) f\left(\frac{a \tau+b}{c \tau+d}\right)=0
$$

we have (under the natural assumption that $f$ and $f^{\prime}$ are independent)

$$
A\left(\frac{a \tau+b}{c \tau+d}\right)=(c \tau+d)^{2} A(\tau)-\frac{k+1}{\pi i} c(c \tau+d)
$$

and

$$
B\left(\frac{a \tau+b}{c \tau+d}\right)=(c \tau+d)^{4} B(\tau)-\frac{k}{2 \pi i} c(c \tau+d)^{3} A(\tau)+\frac{k(k+1)}{(2 \pi i)^{2}} c^{2}(c \tau+d)^{2}
$$


By the quasimodular property of $E_{2}(\tau)$ (recalled in $\$ 3$ ), the equation (2) says that $A(\tau)+(k+1) E_{2}(\tau) / 6$ transforms like a modular form of weight 2 under the action of $\mathrm{SL}_{2}(\mathbb{Z})$. By our assumption that $A(\tau)$ is holomorphic on $\mathfrak{H}$ and bounded when $\Im(\tau) \rightarrow \infty$, this must be 0 and hence

$$
A(\tau)=-\frac{k+1}{6} E_{2}(\tau) .
$$

Putting this into (2) and using

$$
E_{2}^{\prime}\left(\frac{a \tau+b}{c \tau+d}\right)=(c \tau+d)^{4} E_{2}^{\prime}(\tau)+\frac{1}{\pi i} c(c \tau+d)^{3} E_{2}(\tau)+\frac{3}{(\pi i)^{2}} c^{2}(c \tau+d)^{2},
$$

we conclude that $B(\tau)-k(k+1) E_{2}^{\prime}(\tau) / 12$ behaves like a modular form of weight 4 on $\mathrm{SL}_{2}(\mathbb{Z})$. Hence, we have

$$
B(\tau)=\frac{k(k+1)}{12} E_{2}^{\prime}(\tau)+\alpha E_{4}(\tau)
$$

with some constant $\alpha$. But an easy calculation shows that if $f(\tau)$ is a solution of

$$
f^{\prime \prime}(\tau)-\frac{k+1}{6} E_{2}(\tau) f^{\prime}(\tau)+\left(\frac{k(k+1)}{12} E_{2}^{\prime}(\tau)+\alpha E_{4}(\tau)\right) f(\tau)=0,
$$

then $f(\tau) \Delta(\tau)^{\beta}$ is a solution of $(\#)_{k^{\prime}}$ with $\beta$ a solution of

$$
\beta^{2}+\frac{k+1}{6} \beta+\alpha=0
$$

and $k^{\prime}=k+12 \beta$. Hence we may take $\alpha=0$ without losing any generality and we therefore conclude that $(\#)_{k}$ is essentially unique.

Remark. The above quadratic equation for $\beta$ has no real solution when

$$
\left(\frac{k+1}{6}\right)^{2}-4 \alpha<0
$$

However, if the equation (5) has a power series solution starting with $q^{m}+\cdots$, then by equating the coefficient of $q^{m}$ we should have the relation

$$
m^{2}-\frac{k+1}{6} m+\alpha=0 .
$$

Thus, if the inequality (6) holds, no power series solution to (5) can exist.

If we loosen the condition posed on the coefficients of (1), we get a variety of similar differential equations. It may be an interesting task to seek for modular or quasimodular solutions of those equations and study their properties. 
The $\mathrm{SL}_{2}(\mathbb{Z})$-invariance property of solutions of $(\#)_{k}$ has an interesting consequence concerning the space of solutions when $k \equiv 5 \bmod 6$ (quasimodular case) and the representation of $\mathrm{SL}_{2}(\mathbb{Z})$ on this space.

We first observe that the quasimodular solution in Theorem 2 can be written in the form

$$
f(\tau) E_{2}(\tau)+g(\tau)\left(=: F_{k}(\tau)\right)
$$

where $f(\tau)$ and $g(\tau)$ are $\mathrm{SL}_{2}(\mathbb{Z})$-modular of weight $k-1$ and $k+1$ respectively. (We have used $E_{4}^{\prime}=\left(E_{4} E_{2}-E_{6}\right) / 3$.) Write the action of $\mathrm{SL}_{2}(\mathbb{Z})$ of weight $k$ on a function $F$ by

$$
\left.F\right|_{k} \gamma:=(c \tau+d)^{-k} F\left(\frac{a \tau+b}{c \tau+d}\right) \quad \text { for } \quad \gamma=\left(\begin{array}{ll}
a & b \\
c & d
\end{array}\right) \in \mathrm{SL}_{2}(\mathbb{Z}) .
$$

By the transformation formula

$$
E_{2}\left(\frac{a \tau+b}{c \tau+d}\right)=(c \tau+d)^{2} E_{2}(\tau)+\frac{6}{\pi i} c(c \tau+d),
$$

we have

$$
\begin{aligned}
& \left.F_{k}(\tau)\right|_{k}\left(\begin{array}{ll}
a & b \\
c & d
\end{array}\right) \\
& =(c \tau+b)^{-k}\left((c \tau+b)^{k-1} f(\tau)\left((c \tau+b)^{2} E_{2}(\tau)+\frac{6}{\pi i} c(c \tau+b)\right)+(c \tau+b)^{k+1} g(\tau)\right) \\
& =(c \tau+b)\left(f(\tau) E_{2}(\tau)+g(\tau)\right)+\frac{6}{\pi i} c f(\tau) \\
& =c\left(\tau F_{k}(\tau)+\frac{6}{\pi i} f(\tau)\right)+d F_{k}(\tau) .
\end{aligned}
$$

Hence we see that the function

$$
\tau F_{k}(\tau)+\frac{6}{\pi i} f(\tau)
$$

is also a solution of $(\#)_{k}$ and the space of solutions is spanned by this and the $F_{k}(\tau)$. Moreover, the action of $\mathrm{SL}_{2}(\mathbb{Z})$ on this function is computed as

$$
\begin{aligned}
& \left.\left(\tau F_{k}(\tau)+\frac{6}{\pi i} f(\tau)\right)\right|_{k}\left(\begin{array}{ll}
a & b \\
c & d
\end{array}\right) \\
& =\frac{a \tau+b}{c \tau+d}\left(\left.F_{k}(\tau)\right|_{k}\left(\begin{array}{ll}
a & b \\
c & d
\end{array}\right)\right)+\frac{6}{\pi i}(c \tau+d)^{-1} f(\tau) \\
& =\frac{a \tau+b}{c \tau+d}\left((c \tau+d) F_{k}(\tau)+\frac{6}{\pi i} c f(\tau)\right)+\frac{6}{\pi i}(c \tau+d)^{-1} f(\tau) \\
& =(a \tau+b) F_{k}(\tau)+\frac{6}{\pi i} a f(\tau) \\
& =a\left(\tau F_{k}(\tau)+\frac{6}{\pi i} f(\tau)\right)+b F_{k}(\tau) .
\end{aligned}
$$


This, together with (7), shows that the representation of $\mathrm{SL}_{2}(\mathbb{Z})$ on the space of solutions of $(\#)_{k}$ in the case $k \equiv 5 \bmod 6$ is faithful and in particular identical if we choose $\tau F_{k}(\tau)+6(\pi i)^{-1} f(\tau)$ and $F_{k}(\tau)$ as a basis.

As a corollary, we conclude that when $k \equiv 5 \bmod 6$, no modular solution of $(\#)_{k}$ of weight $k$ on any subgroup of $\mathrm{SL}_{2}(\mathbb{Z})$ exists because otherwise there would exist a subspace invariant under the action of that group. As for the other cases, we have no proof that the solutions presented in Theorem 1 exhaust all possible modular solutions (at least when $k$ is an integer or half an integer) but conjecture so on the basis of numerical experiments. As mentioned in the end of Section 3, it would be desirable to have, if any, a number theoretical reason for the existence of modular solutions.

\section{BIBLIOGRAPHY}

1. M. Kaneko and D. Zagier, Supersingular $j$-invariants, Hypergeometric series, and Atkin's orthogonal polynomials, AMS/IP Studies in Advanced Mathematics 7 (1998), 97-126.

2. Ikuo Satake, Flat structure for the simple elliptic singularity of type $\tilde{E}_{6}$ and Jacobi form, Proc. of the Japan Academy 69, Ser. A, No. 7 (1993), 247-251.

3. Ikuo Satake, Flat structure and the prepotential for the elliptic root system of type $D_{4}^{(1,1)}$, in Topological Field Theory, Primitive Forms and Related Topics (Kashiwara, Matsuo, Saito, and Satake eds.), Progress in Math. 160 (1998), 427-452. 